\title{
PROVA ORGANOLÉPTICA COM CARNES BUBALINAS E BOVINAS DE ANIMAIS CRIADOS NAS PASTAGENS DE VÁRZEAS DA AMAZÔNIA CENTRAL
}

\author{
Jörg J. OHLY ${ }^{1}$
}

RESUMO-Devido ao domínio do mercado e do consumo pela carne bovina, aos hábitos de consumo tradicionais e à qualidade inferior da oferta de carne bubalina (Bubalus bubalis), esta ainda é rejeitada ou pelo menos é considerada de qualidade inferior em muitos países, assim como também no Brasil. Para verificar se há uma clara rejeição a carne bubalina devido a de critérios tais como sabor, aroma, maciez, textura, suculência, cor da gordura, cor da carne e aceitação geral, foi realizada uma prova organoléptica na cidade de Manaus-AM, Brasil. Em um churrasco tradicional foram comparados cortes habituais de carne bubalina e bovina. Os resultados mostraram que ambos os tipos de carne tinham uma qualidade semelhante e que são infundados os preconceitos existentes a respeito da carne bubalina.

Palavras-chave: Carne bubalina, carne bovina, prova organoléptica, várzea, Amazônia, Brasil

Organoleptic Assessment of Water Buffalo Meat and Beef of Animals Raised on Central Amazonian Floodplain Pastures

ABSTRACT - Owing to the dominance of the beef market, traditional consumer habits and the often inferior quality of water buffalo (Bubalus bubalis) meat offered, the meat of this species is still rejected in many countries, like it is the case of Brazil, or at least considered as low-grade meat. In order to find out whether or not there is a distinct rejection of water buffalo meat on account of criteria such as taste, flavour, tenderness, texture, juiciness, colour of fat, colour of meat and general acceptability, an organoleptic test was organized in the city of Manaus-AM, Brazil. Traditionally barbecued (churrasco) Brazilian-style cuts of water buffalo meat and beef were compared. The result showed that both types of meat have a comparable quality and that the still existing prejudices in respect of water buffalo meat are unfounded.

Keywords: water buffalo meat, beef, organoleptic test, floodplains, Amazonia, Brazil

\section{INTRODUÇÃO}

A carne bubalina é considerada, em geral, inferior a carne bovina e tem baixa aceitação em muitos países. Há várias razões para isso. Em países como a India, Sri Lanka, China, no sudeste asiático e em regiões a leste do mar Mediterrâneo os búfalos (Bubalus bubalis) só são abatidos quando não servem mais para a tração, a reprodução ou a produção de leite, atingindo assim uma idade superior a 10 anos ou mais, tendo portanto a carne uma qualidade inferior (MATSUKAWA et al., 1976;
YADAVA \& SINGH, 1974). Deve-se levar em conta, também, os preconceitos, principalmente em países com uma longa tradição na criação de gado e com o respectivo hábito de consumo, como é o caso do Brasil. Ironicamente, no entanto, em estados como o Pará e o Amazonas parte dos produtos cárneos oferecidos nos mercados locais, são declarados como carne bovina, quando na realidade, sem o conhecimento do consumidor, é de origem bubalina. No estado do Amazonas a cota é estimada em $15 \%$ (HUND, 1995; OHLY \& HUND,

\footnotetext{
Max-Planck-Institut für Limnologie Postfach 165, 24302 Plön, Alemanha
} 
1996). O interesse em integrar o búfalo na criação de gado tradicional no Brasil fica cada vez mais claro através da multiplicidade de trabalhos cientificos sobre a criação, reprodução, alimentação e saúde dessa espécie nos últimos tempos (e.g. MÜLLER et al., 1994; NASCIMENTO et al., 1979; OHLY, 1986; MÜNCHOW \& PISARZ, 1994; VALE, 1994). Principalmente o trabalho da EMBRAPA-CPATU em Belém e Santarém no estado do Pará (cf. WBC, 1994) evidencia a importância dada ao búfalo para o desenvolvimento das áreas alagáveis da região oriental da bacia amazônica. Apesar do búfalo, o qual está adaptado a um tipo de vida semi-aquático, ser economicamente mais vantajoso do que as raças bovinas de alta produção e também mais vantajoso do que o gado crioulo (raça bovina mixta com um grau de sangue zebuíno alto) das regiões alagadas do Amazonas, o qual está adaptado as condições climáticas e ao valor nutritivo baixa das pastagens regionais (HUND, 1995; OHLY, 1986; OHLY \& HUND, 1996), há ainda poucas noções específicas sobre o búfalo por parte da indústria de carne, dos restaurantes e do consumidor. Nas fazendas das várzeas a situação é outra. Embora a cota de búfalos na criação de animais de grande porte seja apenas de $4 \%$, os rebanhos dos municípios de Careiro, Parintins e Autazes cresceram de fator 11 ate 17 nos anos 1980-1991. Nesse mesmo espaço de tempo a população de búfalos no estado do Amazonas aumentou oito vezes o seu efetivo populacional (IBGE, 1991; OHLY \& HUND, 1996).

$\mathrm{Na}$ verdade há pouca diferença entre a carne bubalina e a bovina, e o consumidor comum quase não nota a diferença. Isso se confirma através de análises físicas e químicas e provas organolépticas realizados em vários países do mundo, que comparam búfalos de "tipo rio" (aos quais pertencem as raças reconhecidas oficialmente no Brasil Mediterrâneo ou Preto, Murrah e Jafarabadi) e o "tipo pântano" (representado no Brasil pela raça Carabao) com raças das espécies bovinas Bos taurus e Bos indicus (e.g. BORGHESE et al., 1978; FERRARA \& INFASCELLI, 1994; FRANCISCIS \& MORAN, 1992; JOHNSON \& CHARLES, 1975; MÜLLER et al., 1991; 1994; ROBERTSON et al., 1982; ROBERTSON et al., 1986; VALIN et al., 1984; YADAVA \& SINGH, 1974). Em virtude do alto conteúdo de pigmentos e mioglobina a cor da carne é mais escura do que a bovina, tendo a gordura branca, com a percentagem de gordura intramuscular menor, a quantidade de gordura separável maior e a percentagem de colesterol menor (comparando-se animais que tiveram o mesmo tipo de criação e alimentação).

Fatores determinantes para o lançamento eficaz de novos produtos de carne no mercado são: aceitação do produtor, produtividade e rentabilidade na produção, emprego da carne e aceitação do consumidor. A entrada da carne bubalina no mercado brasileiro ocorreu de modo discreto, sob o domínio do mercado de carne bovina. Em todos os casos para o brasileiro "carne" é sinônimo de carne de gado. Durante muito tempo as estatísticas oficiais não separavam a carne 
bubalina e bovina. Ainda hoje isso é comum. Visto que desde o início o búfalo foi usado apenas como complemento para o boi e não como uma substituição em regiões inadequadas para o gado e como uma espécie de características próprias de aproveitamento (carne, leite e tração), tanto produtor como indústria não estão suficientemente preparados para as necessidades e particularidades dessa espécie, e o consumidor está pouco informado sobre esse tipo de carne.

A rentabilidade da criação de búfalos nas várzeas do estado do Amazonas em comparação direta com a pecuária bovina tradicional foi comprovada por HUND (1995). O que ainda falta é aceitação da carne bubalina, como também do leite e de seus derivados. Tanto os fazendeiros, matadouros e açougueiros dizem que, em geral, a carne bubalina é de pouco rendimento e de difícil venda em comparação com a carne bovina. Não há uma demanda especifica no que diz respeito a esses produtos, e também não poderia haver, pois o consumidor é mal informado. Até o presente momento não foram feitas, no estado do Amazonas, campanhas publicitárias para a carne bubalina assim como na Austrália, Itália e em menores proporções nos Estados Unidos. Vale salientar, que até então não havia necessidade para isso, pois esses produtos puderam ser vendidos como carne bovina, face a possivel desvalorização imposta indevidamente. Mas, devido ao aumento crescente do sistema de produção e maior conhecimento, esses derivados deverão se impor no mercado. Isso também estaria de acordo com as determinações legais, pois o búfalo é uma espécie domesticada já aceita a nível regional como o boi, a cabra, a ovelha ou o porco.

Há uma série de métodos físicos e químicos reconhecidos que, em regra geral, servem para avaliar a qualidade da carne (BOUTON et al., 1975; JOKSIMOVIC, 1969; JOKSIMOVIC \& OGNJANOVIC, 1977) sendo esses métodos mais significativos para a composição e utilização industrial da carne. 0 consumidor atua subjetivamente, suas prefêrencias são determinadas pelos órgãos dos sentidos e pelo hábito, havendo contudo um receio natural ao novo, não costumeiro.

Para por em andamento uma ampla discussão sobre a qualidade da carne bubalina em comparação direta com a bovina foi realizada uma prova de degustação no Instituto Nacional de Pesquisas da Amazônia (INPA) em Manaus, Amazonas. Além dos degustadores foram convidados mais de 100 funcionários do instituto e a mídia local (imprensa e televisão). Testes semelhantes, numa qualidade de exames e demonstrações independentes, mas também como complemento de análises quimicas e físicas, já foram realizados em muitos paises. Como por exemplo na Austrália(CHARLES, 1982; ROBERTSON et al., 1982; ROBERTSON et al., 1986), Trinidade e Tobago (WILSON, 1961), Sri Lanka (MATSUKAWA et al., 1976), e na Itália (BORGHESE et al., 1978). Mas também em estados brasileiros como o Rio Grande do Sul (MÜLLER et al., 1991; 1994) e o Pará (NASCIMENTO et al., 1978). 


\section{MATERIAL E MÉTODOS}

A carne para o teste organoléptico foi fornecida pelo matadouro estadual FRIGOMASA de Manaus. Ambos animais a serem testados provinham de fazendas de criação localizadas nas regiões alagadas das redondezas de Manaus, eram do sexo masculino, não eram castrados, e tinham um peso vivo de $350 \mathrm{~kg}$ bubalino e $250 \mathrm{~kg}$ bovino respectivamente. $\mathrm{O}$ bovino foi criado num pasto artificial (Brachiaria radicans, capim de água/capim de brejo) e enquanto o bubalino em pastagens nativas, ricas em macrófitas herbáceas de alto valor nutritivo (OHLY, 1986; 1987). A idade do bovino era de 25 meses e a do búfalo de 24 meses. Esse tipo de criação e os parâmetros de rendimento são típicos das várzeas ao redor de Manaus (HUND, 1995; OHLY, 1986; OHLY \& HUND, 1996), no entanto, o bovino ainda não havia atingido o peso médio de abate de $350-360 \mathrm{~kg}$. Devido a uma engorda mais lenta o bovino somente atinge um peso comparável após a idade de 36 meses. A engorda média do bovino, portanto, leva aproximidamente 12 meses mais do que a o bubalino. Como a quantidade de gordura e de tecido conjuntivo na carne aumenta com a idade e, consequentemente sua qualidade diminui, foram escolhidos dois animais da mesma idade.

Ambos animais não eram de raça pura, o que corresponde a situação real nas fazendas de várzea da Amazônia Central. $\mathrm{O}$ bovino era um cruzamento de várias raças de zebu, enquanto que no bubalino predominava fenotipicamente a raça Mediterrâneo, que é uma raça de dupla aptidão (carne e leite), entretanto no Brasil, tem servido em primeiro lugar para a produção de carne (OHLY, 1986).

Antes de ser utilizada, a carne de ambos animais ficou no frigorifero durante 24 horas para um processo de resfriamento (chilling). Na prática isso quase nunca acontece, contudo o tratamento têm um efeito significativo na maciez da carne. Antes do teste houve o retalhamento da carne em cortes típicos brasileiros como picanha, filé, contra-filé, alcatra, lagarto e costela.

A forma escolhida de servir a carne foi o churrasco, preparada com sal grosso aproximidamente uma hora antes de ser assada, com os pedaços assados inteiros em uma grelha sobre carvão ardente, com a distância entre o carvão e a carne de $80 \mathrm{~cm}$. O grau de cozimento da carne foi "bem passada".

Em pratos marcados com A e B foram servidas aos degustadores respectivamente carne bubalina ou bovina, com distribuição dos dois tipos de carne ocorrendo de modo casual.

Foram convidados ao todo 15 degustadores, cuja tarefa consistia em julgar a carne oferecida nos pratos marcados com A e B auxiliados por uma escala de avaliação, com os valores que iam de $1-5$, sendo que o 1 era o valor mais baixo (não aceitável) e o 5 o valor mais alto (muito bom, delicioso), tendo sido adotados os seguintes critérios de julgamento: sabor, aroma, maciez, textura, suculência, cor da gordura, cor da carne e aceitação geral.

Em virtude dos 8 critérios de 
julgamento e da nota máxima 5 , cada corte podia atingir no máximo 40 pontos. Para a avaliação foram calculados os valores médios por corte, espécie e critério de julgamento. A adição desses valores médios fornecia o total de pontos que cada corte e espécie atingira.

\section{RESULTADOS}

As tabelas 1 e 2 mostram a média de pontos dada pelos 15 degustadores para os 8 critérios de julgamento e para os 6 cortes de carne de cada espécie. A carne bovina só atingiu um total de pontos maior nos cortes "picanha" $(+7,5$ $\%$ ) e "alcatra" $(+3,0 \%)$, enquanto que o contrafilé da carne bubalina ganhou claramente a preferência com $10,9 \%$. No que diz respeito aos corte "lagarto" $(+2,4 \%)$ e "costela" $(+4,2 \%)$ a carne bubalina ficou um pouco mais acima.

Considerando-se o julgamento dos cortes a respeito de cada critério e levando-se em conta apenas diferenças apartir de 0,4 pontos (isso corresponde a $8 \%$ da avaliação mais alta) obtemse o seguinte resultado:

Picanha: o sabor $(+1,0$ pontos) e a suculência $(0,7)$ da carne bovina são claramente melhor avaliados. No que diz respeito aos outros critérios, como também a aceitação geral, a avaliação é quase a mesma.

Filé: com relação a suculência (+ $0,6)$ e a cor da gordura $(+0,5)$ a carne bubalina ganha a preferência. No tocante aos outros critérios as diferenças foram poucas.

Contra-filé: nesse corte a carne bubalina ganha a preferência em todos os critérios, verificadas nitidamente na suculência $(+0,9)$, sabor $(+0,8)$, aroma $(+0,4)$, cor da gordura $(+0,4)$ e cor da carne $(+0,4)$.

Alcatra: no total a carne bovina ganha a preferência, mas as diferenças ficam todas só por volta dos 0,3 pontos. Lagarto: só a suculência $(+0,5)$

Tabela 1. Prova de degustação: média de pontos $(n=15)$ por parâmetro, total de pontos por corte e vantagens percentuais por corte e espécie.

\begin{tabular}{|c|c|c|c|c|c|c|}
\hline \multirow[b]{2}{*}{ Parâmetros } & \multicolumn{2}{|c|}{ Picanha } & \multicolumn{2}{|c|}{ Filé } & \multicolumn{2}{|c|}{ Contrafilé } \\
\hline & bub & bov & bub & bov & bub & bov \\
\hline Sabor & 3.3 & 4.3 & 4.2 & 4.2 & 4.7 & 3.9 \\
\hline Aroma & 4.2 & 4.4 & 4.3 & 4.3 & 4.4 & 4.0 \\
\hline Maciez & 3.6 & 3.9 & 4.0 & 4.3 & 4.0 & 3.7 \\
\hline Textura & 3.9 & 3.6 & 4.4 & 4.3 & 4.0 & 3.7 \\
\hline Suculência & 2.9 & 3.6 & 4.2 & 3.8 & 4.4 & 3.5 \\
\hline Cor da gordura & 3.4 & 3.5 & 4.2 & 3.7 & 3.8 & 3.4 \\
\hline Cor da carne & 3.8 & 4.0 & 4.4 & 4.3 & 4.3 & 3.9 \\
\hline Aceitação geral & 3.8 & 3.9 & 4.5 & 4.3 & 4.3 & 4.1 \\
\hline Total de pontos & 28.9 & 31.2 & 34.2 & 33.2 & 33.9 & 30.2 \\
\hline Vantagem percentual & & 7.5 & 5.8 & & 10,9 & \\
\hline
\end{tabular}

bub = carne bubalina

bov $=$ carne bovina 
Tabela 2. Prova de degustação: média de ponto. (i i i 5 ) por parâmetro, total de pontos por corte e vantagens percentuais por corte e espécie.

\begin{tabular}{|c|c|c|c|c|c|c|}
\hline \multirow[b]{2}{*}{ Parâmetros } & \multicolumn{2}{|c|}{ Alcatra } & \multicolumn{2}{|c|}{ Lagarto } & \multicolumn{2}{|c|}{ Costela } \\
\hline & bub & bov & bub & bov & bub & bov \\
\hline Sabor & 4.1 & 4.1 & 4.1 & 4.2 & 4.3 & 4.2 \\
\hline Aroma & 4.1 & 4.4 & 4.1 & 4.2 & 4.1 & 3.9 \\
\hline Maciez & 4.1 & 4.0 & 4.1 & 4.0 & 3.7 & 3.9 \\
\hline Textura & 4.0 & 4.0 & 4.1 & 4.0 & 4.4 & 4.4 \\
\hline Suculência & 4.2 & 4.4 & 4.4 & 3.9 & 4.3 & 4.1 \\
\hline Cor da gordura & 4.1 & 4.0 & 4.1 & 4.2 & 3.9 & 3.8 \\
\hline Cor da carne & 4.2 & 4.5 & 4.4 & 4.2 & 4.3 & 3.7 \\
\hline Aceitação geral & 3.9 & 4.2 & 4.4 & 4.2 & 4.6 & 4.2 \\
\hline Total de pontos & 32.6 & 33.6 & 33.7 & 32.9 & 33.6 & 32.2 \\
\hline Vantagem percentual & & 3.0 & 2.4 & & 4.2 & \\
\hline
\end{tabular}

bub = carne bubalina

bov = carne bovina

da carne bubalina foi melhor avaliada, a diferença a respeito dos outros critérios fica por volta dos 0,2 pontos.

Costela: a carne bubalina ganhou uma vantagem considerável nos critérios maciez $(+0,5)$, aroma $(+0,4)$, cor da carne $(+0,4)$ e aceitação $(+0,4)$.

A tabela 3 mostra a média de pontos dos 8 critérios atingida no total pela carne bubalina e bovina em todos os cortes. As diferenças no número de pontos são de apenas 0,1 , com exceção do critério de suculência.

Tabela 3. Prova de degustação: média por parâmetro e espécie de carne.

\begin{tabular}{lcc}
\hline Parâmetros & Carne bubalina & Carne bovina \\
\hline Sabor & 4.1 & 4.2 \\
Aroma & 4.2 & 4.2 \\
Maciez & 3.9 & 4.0 \\
Textura & 4.0 & 4.1 \\
Suculència & 4.2 & 3.8 \\
Cor da gordura & 3.9 & 3.8 \\
Cor da carne & 4.2 & 4.2 \\
Aceitaçăo geral & 4.3 & 4.2 \\
\hline
\end{tabular}

Nessa, a carne bubalina ganha uma vantagem de 0,4 pontos, enquanto o número de pontos atingidos ficam por volta de 3,8 até 4,3, ultrapassando, assim, claramente a avaliação média de 3 pontos.

O parâmetro "aceitação geral" pode ser interpretado como um resumo de todos os outros parâmetros e como uma espécie de julgamento geral. A tabela 4 mostra que nove até doze dos quinze degustadores classificaram os cortes de carne bubalina como superiores ou iguais aos de carne bovina. Apenas três até seis dos

Tabela 4. Avaliação do parâmetro "aceitação geral" da carne bubalina em relação a carne bovina $(n=15)$.

\begin{tabular}{|c|c|c|c|}
\hline Aceitação geral & superior & igual & inferior \\
\hline Picanha & 3 & 6 & 6 \\
\hline Filé & 3 & 9 & 3 \\
\hline Contrafilé & 7 & 2 & 6 \\
\hline Alcatra & 4 & 5 & 6 \\
\hline Lagarto & 11 & & 4 \\
\hline Costela & 6 & 4 & 5 \\
\hline
\end{tabular}


degustadores preferiram claramente os respectivos cortes de carne bovina.

$\mathrm{Na}$ entrega dos formulários um dos degustadores disse ser oriundo da Ilha de Marajó, região importante para a criação de búfalos no Pará, e conhecer bem a carne bubalina. Disse também, poder tido reconhecer a carne bubalina durante toda a degustação e lamenta ter que afirmar, que a qualidade da carne bovina em todos os cortes tenha sido melhor. De fato, no entanto, exatamente esse degustador classificou em seu formulário todos os cortes da came bubalina como superior aos de carne bovina.

Uma informação foi dada à parte pelos churrasqueiros. Segundo eles, os cortes de carne bubalina levaram bem menos tempo para assar, além de dedicarem maior atenção a carne para que não ficasse muito seca. Isso se deve provavelmente a baixa quantidade de gordura intramuscular.

\section{DISCUSSÃO}

Do ponto de vista científico certamente não se deve supervalorizar esse tipo de teste, que é apenas de carater subjetivo, demonstrativo e indicativo. Contudo, o resultado evidente dessa prova organoléptica mostra que a carne bubalina, tanto nos assim chamados cortes nobres (picanha ou filé) como também nos cortes menos nobres, os quais na prática são muito mais usados para 0 churrasco, possui uma qualidade comparável a da carne bovina. Essa afirmação corresponde a pesquisas feitas no Brasil (NASCIMENTO et al., 1978; MÜLLER et al., 1991; 1994) e em outros países (cf. FRANCISCIS \& MORAN,
1992; WILSON, 1961; VALE, 1994).

O bubalino, como animal de engorda, com o mesmo tipo de criação, alimentação, sexo, tempo de engorda e preparo da carne, pode concorrer plenamente com o boi e tem, assim, como produto próprio uma chance no mercado brasileiro.

O fato de que aproximidamente $2 /$ 3 dos degustadores terem classificado os cortes de carne bubalina como superiores,e pelo fato de não terem notado nenhuma diferença (tabela 4), deveria servir de motivação para os produtores, a indústria de carne e o comércio. Para se fazer da produção bubalina no estado do Amazonas como um ramo próprio de produção da economia pecuária, capaz de identidade própria, necessita-se de esforços coletivos, suplementares por parte das autoridades responsáveis pelo setor pecuário, da pesquisa e extensão agrícola e das associações de pecuaristas no que diz respeito a pesquisa aplicada, a potencialidade da mesma, além de se estabelecer um sistema de comercialização adequado desse gênero alimenticio de alto valor. Além disso, o setor privado (fazendas, indústria e comércio de carne) deveria tomar a iniciativa e fazer uma propaganda dos produtos de carne bubalina, lançandolos, assim, de modo eficaz no mercado.

Usar o consumidor com seus preconceitos e seu compreensível receio como álibi para justificar o fato de a carne bubalina não ser vendida no mercado como produto próprio, certamente não soluciona o problema. Se o consumidor não conhece ou só conhece pouco um produto, não pode haver, 
também, uma demanda especifica.

É interessante saber, que em 1995 foi dado um primeiro passo na direção certa. A iniciativa partiu do setor privado. Um açougue em Manaus, a capital do estado do Amazonas e sem dúvida o mercado mais importante na Amazônia Central, oferece desde então intencionalmente a seus clientes carne bubalina e os informa sobre diferenças e vantagens dessa espécie em comparação com a conhecida carne bovina. Contudo, o açougueiro, que é oriundo de Parintins, o centro mais importante de criação de búfalos do estado, confessa ser até agora é pouca a aceitação.

\section{AGRADECIMENTOS}

Agradeço à Associação de Servidores do INPA (ASSINPA) que colocou à disposição no dia 7 de novembro de 1992 sua área de lazer para a realização da prova de degustação. Meu agradecimento especial ao grupo de churrasqueiros gaúchos sob a direção do Sr. Humberto dos Santos Aquino pelo preparo competente da carne e a todos os voluntários que contribuíram para o êxito do evento. O resultado deste trabalho é parte da cooperação científica entre o Instituto Nacional de Pesquisas da Amazônia (INPA) de Manaus-AM, Brasil, e o Instituto MaxPlanck de Limnologia (MPI) de Plön, Alemanha. O projeto "Uso múltiplo das áreas alagáveis (várzeas) na Amazônia Central" (No. 0339372A) foi promovido pelo Ministério Federal de Educação, Ciência, Pesquisa e Tecnologia (BMBF) da Alemanha, pelo Conselho Nacional de Desenvolvimento Cientifico e
Tecnológico $(\mathrm{CNPq})$ do Brasil e pelo Instituto Brasileiro de Meio Ambiente e Recursos Naturais Renováveis (IBAMA) nos anos de 1991 a 1994 no âmbito do programa de pesquisa bilateral "Studies on Human Impact on Forests and Floodplains in the Tropics" (SHIFT). A tradução para o português foi feita por Sra. Mônica Schüler (München, Alemanha).

\section{Bibliografia citada}

BORGHESE, A.; ROMITA, A.; GIGLI, S; DI GIACOMO, A. 1978. Eating quality of buffalo and bovine calves slaughtered at 20-28 and 36 weeks of age. In: DE BOER, H.; MARTIN, J. (ed.). Patterns of Growth and Development in Cattle. Nijhoff, Holland, p267-276

BOUTON, P.E.; FORD, A.L.; HARRIS, P.V.; RATCLIFF, D. 1975. Objective-subjective Assessment of Meat Tenderness. Journal of Texture Studies, 6: 315-328

CHARLES, D.D. 1982. Meat Tenderness and Palatability of Swamp Buffalo and Four Breeds of Cattle. Anim. Prod., 34: 79-84

FERRARA, B; INFASCELLI, F. 1994. Buffalo Meat Production. Proceedings of the IV World Buffalo Congress. Vol. I. 27-30 de junho, 1994, Sào Paulo, Brasil, p122-136

FRANCISCIS, G. DE; MORAN, J.B. 1992. Meat production from river buffaloes. In: TULLOH, N.M. \& J.H.G. HOLMES (eds.). Buffalo Production. Elsevier Science Publishers B.V., Amsterdam, The Netherlands, World Animal Series, C6, p413-419

HUND, M. 1995. Möglichkeiten und Grenzen der landwirtschaftlichen Nutzung der Überschwemmungsgebiete (Várzea) am mittleren Amazonas unter besonderer Berücksichtigung der Viehhaltungs- und Dauerkultursysteme. Wissenschaftsverlag Vauck, Kiel, Alemanha. Tese Publicada de Doutoramento (Universidade de Hohenheim, Stuttgart, Alemanha), 173 + VIp. 
IBGE 1991. Anuário Estatístico do Brasil. Rio de Janeiro-RJ, Brasil.

JOHNSON, E.R.; CHARLES, D.D. 1975. Comparisons of Liveweight Gain and Changes in Carcass Composition Between Buffalo (Bubalus bubalis) and Bos taurus Steers. Aust. J. Agric. Res., 26: $415-22$

JOKSIMOVIC, J.S. 1969. Physical, chemical and structural characteristics of buffalo meat. J. Scient. Agric. Res., Belgrade, 22(78): $110-151$

JOKSIMOVIC, J.S.; OGNJANOVIC, A. 1977. Comparison of carcass yield, carcass composition and quality characteristics of buffalo meat and beef. Meat Science, 1: 105-110

MATSUKAWA, T:; TILAKARATNE, N, BUVANENDRAN, V. 1976. Growth and Carcass Characteristics of Cattle and Buffalo Breeds Reared on a Dry Zone Pasture in Sri Lank (Ceylon). Trop. Anim. Hith Prod, 8: 155-162

MÜLLER, L.; AGUIRRE, L.F.; RESTLE, J.; PEROBELLI, Z. 1991. Carcass and Meat Quality of Cattle and Buffalo (Bubalus bubalis). In: Anon (ed.). International Congress of Meat Science and Technology. Proceedings, (1:37), p165-169

MÜLLER, L.; AGUIRRE, L.F.; FEIJO, G.L.D.; PEROBELLI, Z. 1994. Buffalo Meat Quality When Submitted to Three Feeding Regimes. Proceedings of the IV World Buffalo Congress. Vol II. 27 30 de junho, 1994, São Paulo, Brasil, p $127-129$

MÜNCHOW, G;; PISARZ, M. 1994. Epidemiological Studies of Some Economical Significant Infectious Diseases in Water Buffaloes in the Central Amazon Region, Brazil. Proceedings of the IV World Buffalo Congress. Vol II. 27-30 de junho, 1994, São Paulo, Brasil, p353-355

NASCIMENTO, C.N.B.; NETO, M.S.; CARVALHO, L.O.D.M. 1978. Provas de Degustão com Carnes Bovinas e Bubalinas. Anais da 15. Reuniào Anual da Soc, Bras. Zootec., Belém-PA, Brasil: p149
NASCIMENTO, C.N.B.; CARVALHO, L.O.D.M.; LOURENÇO JUNIOR, J.B. 1979. Importância do búfalo para a pecuária brasileira. EMBRAPACPATU, Belèm-PA, Brasil, 32 p.

OHLY, J.J. 1986. Water-Bufallo Husbandry in the Central Amazon Region in View of Recent Developments. Animal Research and Development, $24: 23-40$

OHLY, J.J. 1987. Untersuchungen über die Eignung der natürlichen Pflanzenbestände auf den Überschwemmungsgebieten (Värzea) am mittleren Amazonas, Brasilien, als Weide für den Wasserbüffel (Bubalus bubalis) während der terrestrischen Phase des Ökosystems. Göttinger Beiträge zur Landund Forstwirtschaft in den Tropen und Subtropen, Vol 24, 53-79

OHLY, J.J.; HUND, M. 1996. Pasture Farming on the Floodplains of Central Amazonia Animal Research and Development, 43/44: $27 \mathrm{p}$.

ROBERTSON, J.; BOUTON, P.E.; HARRIS, P.V.; MACFARLANE, J.J.; SHORTHOSE, W.R. 1982. PressureHeat Treatment of Meat: A Comparison of Beef and Buffalo Meat. Meat Science, 10: 285-292

ROBERTSON, J.; RATCLIFF, D.; BOUTON, P.E.; HARRIS, P.V.; SHORTHOSE, W.R. 1986. A Comparison of Some Properties of Meat from Young Buffalo (Bubalus bubalis) and Cattle. Journal of Food Science, 51(1): 47-50

VALE, W.G. 1994. Prospects of Buffalo Production in Latin America. Anon (ed). Proceedings of the $1 \mathrm{~V}$ World Buffalo Congress. Vol 1. 27-30 de junho, 1994, São Paulo, Brasil, p75-87

VALIN, C.; PINKAS, A.; DRAGNEV, H.; BOIKOVSKI, S.; POLIKRONOV, D. 1984. Comparative study of buffalo meat and beef. Meat Science, 10: 69-84

WBC 1994. Proceedings of the IV World Buffalo Congress. Vol 3. Congresso Mundial de Criadores de Búfalos, 27-30 de junho, 1994, São Paulo, Brasil, 363 p. 
WILSON, P.N. 1961. Palatability of Water Buffalo Meat. J. Agric. Soc. Trin and Tob., 61: 457-460

YADAVA, B.S.; SINGH, L.N, 1974, Chemical composition of buffalo meat available from the local slaughterhouse: Indian J. Anim. Sci., 44(10): 746-749 